Dedicated to Prof. Hong-Kun Xu on the occasion of his $60^{\text {th }}$ anniversary

\title{
Inexact descent methods for convex minimization problems in Banach spaces
}

\section{SimEON REICH and AlEXANDER J. ZASLAVSKI}

\begin{abstract}
.
Given a Lipschitz and convex objective function of an unconstrained optimization problem, defined on a Banach space, we revisit the class of regular vector fields which was introduced in our previous work on descent methods. We study, in particular, the asymptotic behavior of the sequence of values of the objective function for a certain inexact process generated by a regular vector field when the sequence of computational errors converges to zero and show that this sequence of values converges to the infimum of the given objective function of the unconstrained optimization problem.
\end{abstract}

\section{INTRODUCTION}

Acknowledgments. The first author was partially supported by the Israel Science Foundation (Grant No. 820/17), by the Fund for the Promotion of Research at the Technion and by the Technion General Research Fund. Both authors are grateful to the two referees for their useful comments and helpful suggestions.

\section{REFERENCES}

[1] Bačák, M., A variational approach to stochastic minimization of convex functionals, Pure Appl. Funct. Anal., 3 (2018), 287-295

[2] Cegielski, A., A method of projection onto an acute cone with level control in convex minimization, Math. Program., 85 (1999), 469-490

[3] Curry, H. B., The method of steepest descent for nonlinear minimization problems, Quarterly Appl. Math., 2 (1944), 258-261

[4] Gibali, A., Küfer, K.-H., Reem, D. and Süss, P., A generalized projection-based scheme for solving convex constrained optimization problems, Comput. Optim. Appl., 70 (2018), 737-762

[5] Grad, S.-M. and Pop, E.-L., Vector duality for convex vector optimization problems by means of the quasi-interior of the ordering cone, Optimization, 63 (2014), 21-37

[6] Griva, I., Convergence analysis of augmented Lagrangian-fast projected gradient method for convex quadratic problems, Pure Appl. Funct. Anal., 3 (2018), 417-428

[7] He, H., Hou, L. and Xu, H.-K., A partially isochronous splitting algorithm for three-block separable convex minimization problems, Adv. Comput. Math., 44 (2018), 1091-1115

[8] Hiriart-Urruty, J.-B. and Lemaréchal, C., Convex analysis and minimization algorithms, Springer, Berlin, 1993

[9] $\mathrm{Hu}, \mathrm{Y} ., \mathrm{Li}, \mathrm{C}$. and Yang, X., On convergence rates of linearized proximal algorithms for convex composite optimization with applications, SIAM J. Optim., 26 (2016), 1207-1235

[10] Konnov, I., On convergence properties of a subgradient method, Optim. Methods Softw., 18 (2003), 53-62

Received: 22.10.2019; In revised form: 12.01.2020; Accepted: 19.01.2020

2010 Mathematics Subject Classification. 49M37, 90C25, 90C30.

Key words and phrases. Banach space, complete metric space, convex function, descent method, inexact iterate, iterative process.

Corresponding author: Simeon Reich; sreich@technion.ac.il 
[11] Mordukhovich, B. S., Variational analysis and generalized differentiation. I: Basic theory, Springer, Berlin, 2006

[12] Polyak, R., Exterior distance function, Pure Appl. Funct. Anal., 2 (2017), 369-394

[13] Reich, S. and Zaslavski, A. J., Generic convergence of descent methods in Banach spaces, Math. Oper. Research, 25 (2000), 231-242

[14] Reich, S. and Zaslavski, A. J., The set of divergent descent methods in a Banach space is $\sigma$-porous, SIAM J. Optim., 11 (2001), 1003-1018

[15] Reich, S. and Zaslavski, A. J., Regular vector-fields in Banach spaces, Taiwanese J. Math., 12 (2008), 11651176

[16] Reich, S. and Zaslavski, A. J., Genericity in nonlinear analysis, Developments in Mathematics, 34, Springer, New York, 2014

[17] Reich, S. and Zaslavski, A. J., Descent methods with computational errors in Banach spaces, Optimization, 14 (2019), 41-50

[18] Reich, S. and Zaslavski, A. J., Inexact descent methods with convex objective functions in Banach spaces, J. Nonlinear Convex Anal., 20 (2019), 1875-1882

[19] Zălinescu, C., Convex analysis in general vector spaces, World Scientific, New Jersey- London-SingaporeHong Kong, 2002

[20] Zălinescu, C., On Lagrange multipliers in convex entropy minimization, Pure Appl. Funct. Anal., 3 (2018), 393-402

[21] Zaslavski, A. J., Optimization on metric and normed spaces, Springer, New York, 2010

[22] Zaslavski, A. J., Numerical optimization with computational errors, Springer, Cham, 2016

DEPARTMENT OF MATHEMATICS

THE TECHNION - ISRAEL INSTITUTE OF TECHNOLOGY

32000 HAIFA, ISRAEL

Email address: sreichetechnion.ac.il

Email address: ajzasletechnion.ac.il 